\title{
Avaliação do Uso de Técnicas FT-IR para Caracterização de Cobertura Polimérica de Material Energético
}

\author{
Elizabeth C. Mattos \\ Instituto de Aeronáutica e Espaço, ITA, CTA \\ Rita C. L. Dutra; Milton F. Diniz \\ Instituto de Aeronáutica e Espaço, CTA \\ Kohun Iha \\ Instituto Tecnológico da Aeronáutica, CTA
}

\begin{abstract}
Resumo: Técnicas FT-IR de transmissão, reflexão e fotoacústica (PAS) foram utilizadas para a caracterização da cobertura polimérica do hexahidro-1,3,5-trinitro-1,3,5-triazina (RDX) usando um poliuretano (Estane). Verificou-se no decorrer deste trabalho que, embora seja possível observar as bandas do polímero por meio das diferentes técnicas, as de transmissão (filme vazado) e fotoacústica foram aquelas que melhor evidenciaram as absorções do poliuretano na amostra analisada da cobertura polimérica do material energético. Entre as técnicas consideradas de análise de superfície, a espectroscopia PAS apresentou melhor resultado, provavelmente em função da espessura de poucos micra da camada polimérica.
\end{abstract}

Palavras-chave: Espectroscopia no infravermelho, polímeros, materiais energéticos.

\section{Evaluation of FT-IR Techniques for Polymeric Coating Characterization of Energetic Material}

Abstracts: The transmission, reflection and photoacoustic FTIR techniques have been used for characterization of polymeric coating of hexahydro-1,3,5-trinitro-1,3,5-triazine (RDX) using a polyurethane (Estane). Although the bands characteristic of the polymer were indicated by the various techniques, the transmission (casting film) and photoacoustic techniques showed better evidence of polyurethane absorptions in the polymer coating of energetic material. Among the surface-sensitive techniques, PAS spectroscopy shows better result, probably because the polymer coating is at few microns from the surface.

Keywords: Infrared spectroscopy, polymer, energetic materials.

\section{Introdução}

Os polímeros usados na obtenção de material altamente energético que recobrem os cristais têm propriedades especiais. São copolímeros que contêm segmentos rígidos e flexíveis. Na obtenção de explosivo plástico (PBX) para produção de cargas prensadas podem ser utilizados polímeros fluorados ou à base de poliuretanos. O produto granular pode ser obtido usando um poliuretano termoplástico, com marca registrada de "Estane", ou polímero como o hexafluorpropileno fluoreto de vinilideno fluorado, conhecido como "Viton"[1]. O Estane, nome dado ao polímero poliuretano termoplástico (TPU) baseado em poliéster aromático preparado por cadeias longas $\mathrm{de}^{[2]} \mathrm{um}$ copolímero de 1,4- butanodiol, de 4,4'-fenilmetileno diisocianato (MDI), e um macrodiol feito do ácido adípico e do 1,4-butanodiol ${ }^{[3]}$. É possível observar a estrutura química da unidade estrutural do poliuretano à base de Estane por meio da Figura 1.

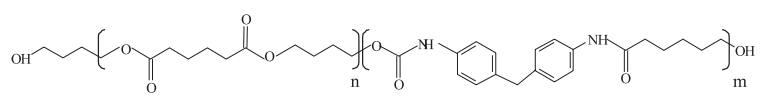

Figura 1. Estane

A constituição dos blocos de Estane pode ser observada na Figura 2, apresentando uma massa molecular média de aproximadamente 2000 .

Este polímero contém dois grupos funcionais os quais são particularmente vulneráveis ao ataque químico: a ligação éster no bloco poliéster e a ligação uretana, formada pela reação do MDI com os componentes diol. Ligação éster em poliuretanos poliéster são, notavelmente, pobres em estabilidade hidrolítica, e a estrutura mostra ser susceptível ao ataque oxidativo.

A quebra de um ou ambos destes tipos de grupos funcionais pode ser a causa da degradação do Estane em armazenamento com explosivo ${ }^{[4]}$. Os explosivos são combinados com polímeros a fim de minimizar sua sensibilidade ao choque, e para que sejam menos perigosos de se manusear.

Autor para correspondência: Elizabeth C. Mattos, Instituto Tecnológico da Aeronáutica, ITA/IAE/ CTA, Praça Marechal do Ar Eduardo Gomes 50, Vila das Acácias, CEP: 12228-904, São José dos Campos, SP. E-mail mattos@directnet.com.br 


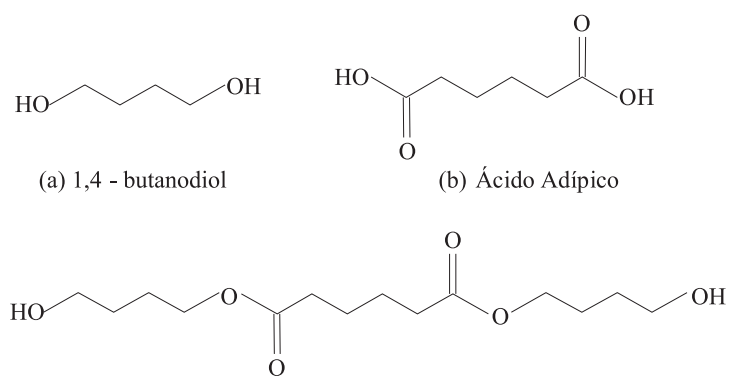

(c) Macrodiol MW 2000

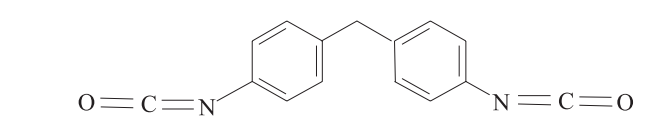

(d) 4,4'- Difenilmetano Diisocianato

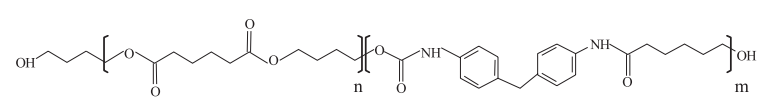

(e) Estane

Figura 2. Esquema de preparação do Estane em blocos

A fim de caracterizar a composição polimérica em explosivo plástico, a espectroscopia no infravermelho com transformada de Fourier (FT-IR) pode ser aplicada. Em princípio, os principais usos da espectroscopia no FT-IR para caracterização de poliuretanos são: teste de classificação para poliuretanos, diferenciação de poliéster para poliéter, verificação da presença de componentes aromáticos com base na absorção em $1600 \mathrm{~cm}^{-1}$ e verificação do uso de água ou diamina na expansão da cadeia polimérica, com base na de absorção em $1640 \mathrm{~cm}^{-1}$.

A amina não é identificada, de modo inequívoco, mas a falta de absorção em 1667-1640 $\mathrm{cm}^{-1}$, entretanto, indica a ausência da diamina ou de água ${ }^{[5]}$.

A presença de estrutura uretânica é detectada com base nas bandas em $1538 \mathrm{~cm}^{-1}$ (amida II - deformação - $\delta \mathrm{NH}$ ), $1724 \mathrm{~cm}^{-1}$ (amida I $-\mathrm{C}=\mathrm{O}$ estiramento $-v$ ) e $3330 \mathrm{~cm}^{-1}(\mathrm{v} \mathrm{NH})$. Ligações poliéster ou poliéter em poliuretanos podem ser detectadas na diferença de intensidade das bandas de absorção em $1250 \mathrm{~cm}^{-1}$ (v COOC) no caso do poliéster-uretano, e por volta de $1110 \mathrm{~cm}^{-1}$ (n COC) para os poliéter-uretano. Poliéster-poliuretanos apresentam uma banda mais forte e larga do que nos poliéteres ${ }^{[5]}$.

A aplicação de diferentes técnicas IR de transmissão, reflexão e fotoacústica para análise na região do infravermelho médio (MIR) é uma boa prática para avaliação mais completa dos materiais ${ }^{[6-8]}$, permitindo a análise de espécies do interior e superfície. Técnicas de detecção fotoacústica (PAS) podem ser utilizadas quando as amostras são de difícil preparo e outras técnicas falham ou fornecem um pequeno número de informações ${ }^{[9]}$.

Em nossos laboratórios, metodologias envolvendo técnicas de transmissão, Microscopia/FT-IR, entre outras de reflexão, e PAS ${ }^{[10,11]}$, têm sido desenvolvidas para caracterização de materiais energéticos.

Diante da importância das coberturas poliméricas na

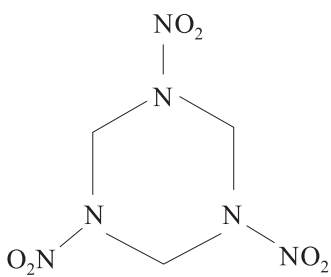

Figura 3. Fórmula estrutural do RDX

obtenção dos explosivos plásticos prensados e de um número pequeno de informações encontradas na literatura sobre caracterização destas coberturas por espectroscopia IR, o presente trabalho tem como objetivo a caracterização por meio de técnicas FT-IR do polímero que está recobrindo o explosivo.

\section{Experimental}

Para realização da cobertura dos cristais de explosivo, tal como RDX (Figura 3) com Estane, a fase líquida inorgânica é a água destilada, onde o explosivo está disperso.

Como fase orgânica, é utilizada uma "laca", ou seja, um plástico dissolvido em solvente orgânico de baixo ponto de ebulição. Este processo é realizado sob forte agitação ${ }^{[2,12]}$. O solvente é removido por destilação sob agitação contínua, onde o plástico precipita-se sobre o explosivo ao final da eliminação do solvente ${ }^{[2]}$. Deste modo foi preparada a amostra da cobertura poliuretânica com RDX, denominada Cobertura 4/91, que foi caracterizada neste trabalho.

Depois de realizado o processo de cobertura, o produto granular à base de RDX, pode ser empregado na obtenção de cargas explosivas prensadas com alto teor energético ${ }^{[1]}$.

As análises FT-IR foram conduzidas com o espectrômetro FT-IR SPECTRUM 2000 PERKINELMER (resolução $4 \mathrm{~cm}^{-1}$; ganho $1 ; 4000$ a $400 \mathrm{~cm}^{-1}, 40$ varreduras). Foram utilizados acessórios de reflexão (reflexão total atenuada - ATR, difusa-DRIFT e microscopia/FT-IR): ATR (região de 4000 a $700 \mathrm{~cm}^{-1}, 26 \%$ de energia, 40 varreduras, sendo que a amostra foi colocada sobre os dois lados do cristal de germânio, $45^{\circ}$ ), DRIFT (região 4000 a $400 \mathrm{~cm}^{-1}, 35 \%$ de energia, 40 varreduras ) e Microscopia/FT-IR (modo reflexão, resolução 8, 128 varreduras). Para a análise PAS, as condições foram: resolução 4 , velocidade $0,05 \mathrm{~cm} / \mathrm{s}, 4000$ a $400 \mathrm{~cm}^{-1}, 32$ varreduras. Os materiais de referência (calibração) utilizados para as análises por ATR, DRIFT, Microscopia-FTIR e PAS foram, respectivamente, cristal de germânio, espelho de calibração, "gold mirror" e negro de fumo.

Para a análise por microscopia, DRIFT e ATR, as amostras foram analisadas tal como recebidas. Para os espectros PAS, as amostras foram diluídas em $\mathrm{KBr}$, em proporção 1:20 $\mathrm{mg} \mathrm{KBr}$, maceradas em gral de ágata e analisadas, tendo como espectro de referência o negro de fumo compactado (padrão MTEC).

As amostras foram preparadas para as análises MIR por meio de diferentes técnicas de transmissão ${ }^{[13]}$ pastilha de $\mathrm{KBr}$ (1,0:400 mg) e como filme vazado (resíduo líquido obtido após tratamento com tetracloreto de carbono e evaporação do solvente).

Filme vazado, também conhecido como "casting" é obtido pela dissolução do polímero em solvente adequado, com 
posterior colocação desta solução em vidro relógio ou célula de $\mathrm{KBr}$ ou $\mathrm{NaCl}$ e evaporação do solvente à sua temperatura de ebulição. Dependendo do material, pode ser obtido um resíduo líquido, viscoso ou sólido.

\section{Resultados e Discussão}

\section{Análise MIR do RDX}

As absorções médias e intensas observadas no espectro FT-IR do RDX (Figura 4), foram escolhidas para caracterizar o composto, em acordo com a literatura ${ }^{[13-15]}$. Uma provável atribuição ${ }^{[13-15]}$ dessas bandas está incluída na Tabela 1.

Algumas abreviações dos modos vibracionais utilizadas são: $v$ - estiramento ou deformação axial, assimétrica $\left(v_{\mathrm{a}}\right)$ e simétrica $\left(v_{\mathrm{s}}\right), \delta$ - deformação angular no plano e $\tau, \gamma$-deformações angulares fora do plano.

\section{Análise MIR do Estane}

A presença de estrutura uretânica é detectada no IR médio ${ }^{[5]}$ com base nas absorções em $1534 \mathrm{~cm}^{-1}(\delta$ do grupo $\mathrm{NH}$ amida II), $1732 \mathrm{~cm}^{-1}$ ( $\vee \mathrm{CO}$-amida I) e $3345 \mathrm{~cm}^{-1}$ ( $(\mathrm{NH})$. Ligações poliéster ou poliéter em poliuretanos podem ser detectadas pela presença da absorção em $1223 \mathrm{~cm}^{-1}$ ( $\mathrm{v}$ grupoCOOC), e $1143 \mathrm{~cm}^{-1}$ ( $v$ COC ) para os poliéter-uretano.

\section{Análise MIR da cobertura polimérica}

É possível observar por meio da Figura 5, que o espectro $\mathrm{A}$, referente à cobertura polimérica, mostra, basicamente, as bandas de absorção para RDX, de acordo com o espectro de referência deste material energético (espectro $\mathrm{B}$, Tabela 1). Entretanto, pode-se observar no espectro A em $1730 \mathrm{~cm}^{-1}$, uma absorção fraca, característica do $\vee \mathrm{C}=\mathrm{O}$, sugerindo a presença de outro material, provavelmente, do polímero que recobre o material energético (espectro D). Este resultado está de acordo com as características da técnica de transmissão, que mostra o que está em maior teor, neste caso, o RDX.

Após tratamento adequado com tetracloreto de carbono, foi obtido um resíduo, o qual foi analisado sob a forma de filme vazado (espectro C) e foi possível, então, comparar este espectro C (resíduo) ao espectro D (espectro referência do Estane), identificando a presença do polímero que recobre o explosivo. As bandas que evidenciam a presença do polímero foram assinaladas nos espectros $\mathrm{C}$ e D com asteriscos.

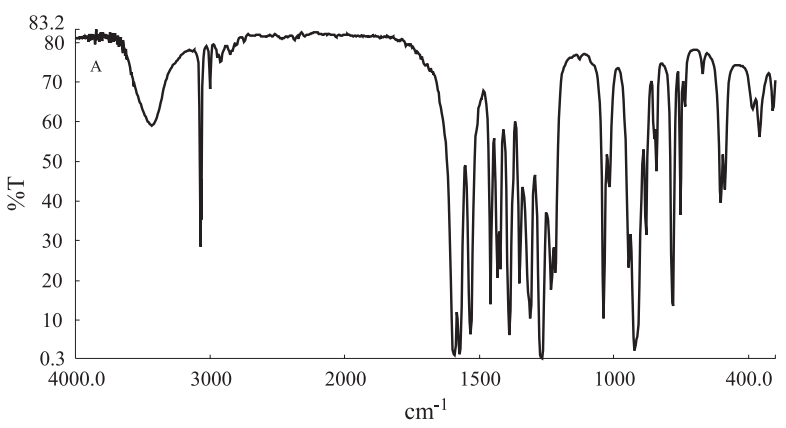

Figura 4. Espectro FT-IR de transmissão do RDX
Tabela 1. Atribuição provável dos grupos funcionais RDX aos modos vibracionais IR correspondentes ${ }^{[15]}$.

\begin{tabular}{cc}
\hline $\begin{array}{c}\text { Número de Onda } \\
\left(\mathbf{c m}^{-1}\right)\end{array}$ & $\begin{array}{c}\text { Modo Vibracional/ } \\
\text { Grupo Funcional }\end{array}$ \\
\hline 3073 & $v \mathrm{CH}_{2}$ \\
1592 & $v_{\mathrm{a}} \mathrm{NO}_{2}$ \\
1459 & $\delta_{\mathrm{s}} \mathrm{CH}_{2}$ \\
1433 & $\delta_{\mathrm{s}} \mathrm{CH}_{2}$ \\
1390 & $\delta_{\mathrm{s}} \mathrm{CH}_{2}$ \\
1351 & $v_{\mathrm{s}} \mathrm{NO}_{2}+v$ N-N \\
1270 & $v \mathrm{~N}-\mathrm{N}+v_{\text {anel }}$ \\
& Vibração do anel \\
1039 & $\delta$ e $\gamma \quad\left(\mathrm{NO}_{2}\right)$ \\
945 & $\tau+\gamma\left(\mathrm{NO}_{2}\right)$ \\
\hline
\end{tabular}

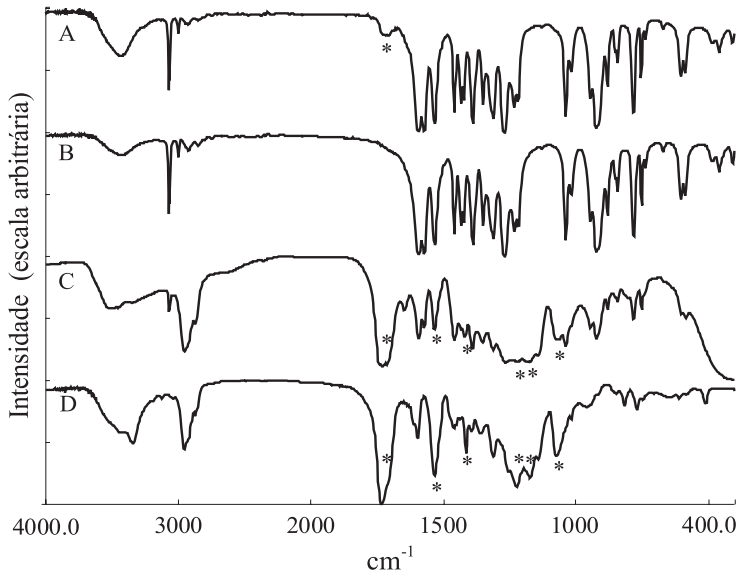

Figura 5. Espectros FT-IR de transmissão de: A) Cobertura 4/91; B) RDX; C) Resíduo obtido após tratamento com tetracloreto de carbono da Cobertura 4/91; D) Estane 5702-F

\section{Análise PAS/Comparação com os dados obtidos por transmissão}

Para melhor entendimento dos resultados obtidos com a análise PAS da cobertura polimérica, o princípio da técnica é descrito a seguir, do mesmo modo que para as outras técnicas de análise de superfície.

A técnica PAS utiliza detecção do sinal acústico de uma amostra gerada por meio de absorção de radiação modulada. A amostra é colocada numa pequena câmara, a qual um microfone é acoplado ${ }^{[16]}$. Radiação modulada é focalizada sobre a amostra, e certas freqüências que correspondem ao espectro de absorção do material são absorvidas. A radiação absorvida causa flutuações de temperatura da superfície.

Estas flutuações da temperatura de superfície da amostra induzem a mudanças periódicas de pressão do gás na célula fotoacústica. Uma onda sonora se desenvolve e é detectada por um microfone. Se uma freqüência particular não é absorvida, então, a amostra não aquecerá e nenhuma onda sonora 


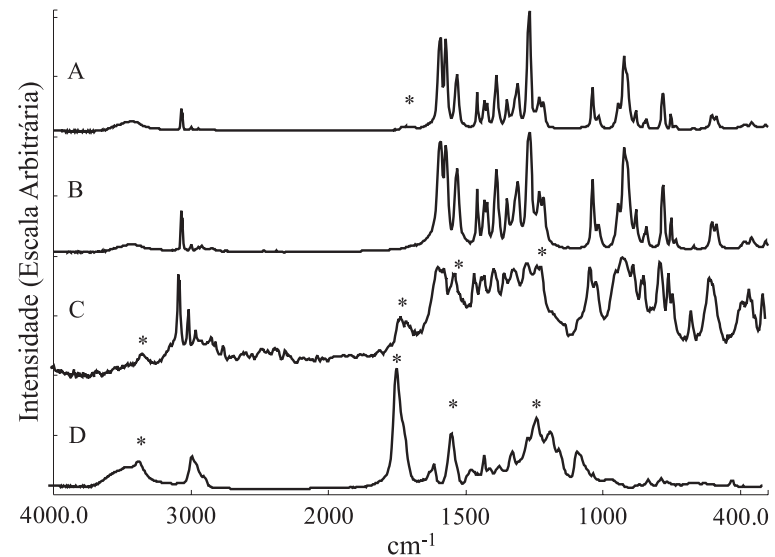

Figura 6. Espectros FT-IR de transmissão e PAS de: A) Cobertura 4/91(transmissão); B) RDX (transmissão); C) Cobertura 4/91 (PAS) ; D) Estane 5702-F (transmissão)

se desenvolverá. Na espectroscopia PAS, ondas sonoras são usadas para detectar freqüências de absorção de infravermelho. Amostras opacas são analisadas sem dificuldades.

Dois processos estão envolvidos na geração do sinal fotoacústico: i) absorção de luz, cuja magnitude é determinada pela propriedades de absorção da amostra; e ii) a liberação de calor para a superfície, este determinado pelas propriedades térmicas da amostra. Como os comprimentos de difusão térmica para a maioria dos materiais orgânicos, na região do infravermelho médio, estão na faixa de algumas dezenas de micra a espectroscopia PAS/FT-IR é considerada uma técnica de análise de superfície próxima (near-surface analysis technique). Na Figura 6 são apresentados os espectros obtidos por PAS e transmissão das amostras de cobertura. É possível observar que o espectro C (PAS/FT-IR) da amostra do material coberto, em comparação com o espectro D de referência do Estane, apresenta absorções do polímero. Portanto, se é possível identificar o polímero usado no processo de cobertura por meio da técnica PAS, com base em suas características, é possível sugerir que este polímero esteja numa camada superficial de poucos micra. Embora no espectro de transmissão (A), seja possível visualizar algumas bandas do polímero, há maior interferência das bandas de RDX. Além disso, o formato das bandas do polímero é melhor visualizado no espectro PAS (C).

\section{Análise por Microscopia-FT-IR/ Comparação com os dados obtidos por transmissão}

A microscopia FT-IR é essencialmente uma técnica para a análise de micro amostras ou áreas específicas de uma amostra $^{[17]}$. O microscópio FT-IR promove o exame visual e documentação da área que foi exposta a radiação IR. Este poder de combinação da imagem visual com o poder analítico da espectroscopia FT-IR confere o sucesso a esta técnica. Ambos os modos, transmissão e reflexão são permitidos. Neste trabalho, o modo reflexão foi utilizado para a análise da cobertura polimérica do material energético. A Figura 7 mostra as micrografias da cobertura polimérica estudada e do material energético RDX.

A comparação das micrografias, obtidas com o microscópio acoplado ao FT-IR, sugere que os cristais de RDX estejam

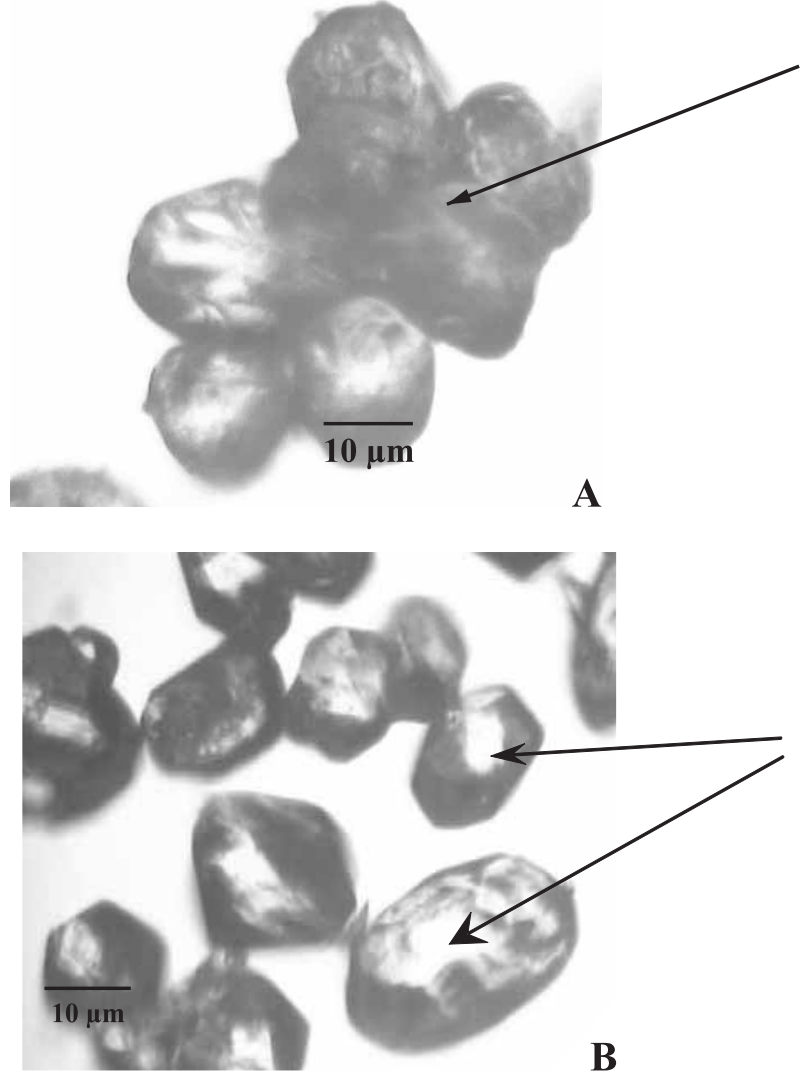

Figura 7. Micrografias FT-IR: (A) da cobertura 04/91 de RDX/Estane, (B) RDX

recobertos com o material polimérico, pois os cristais da micrografia A apresentam redução da reflectibilidade, e estão mais aglomerados em relação aos cristais da micrografia B. Em adição, na análise por espectroscopia IR do material (Figura 8) pode ser observado, que embora a varredura da análise por microscopia FT-IR, em função do detector, seja até $700 \mathrm{~cm}^{-1}$, e o espectro apresente ruídos, é possível visualizar as bandas do Estane (bandas assinaladas). Isto significa que embora a qualidade do espectro não seja tão boa quanto ao obtido

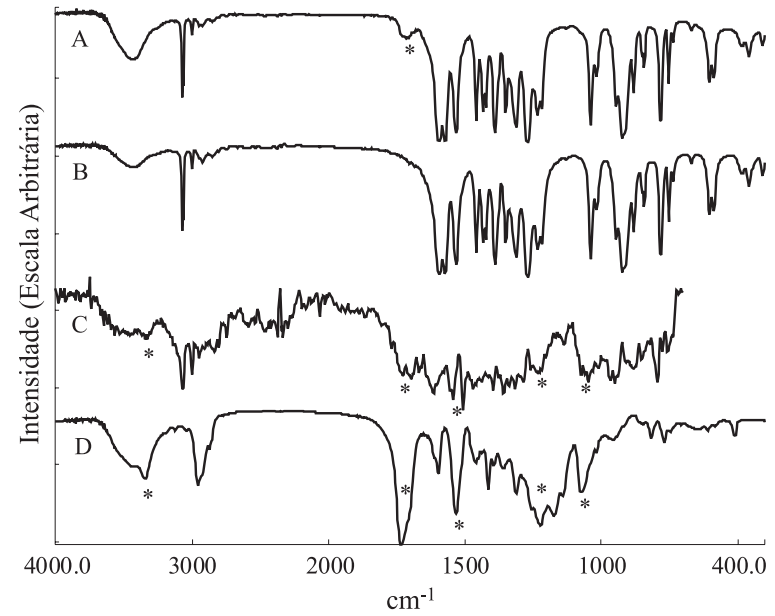

Figura 8. Espectros obtidos por Microscopia-FT-IR e transmissão; A) Cobertura 4/91 (transmissão); B) RDX (transmissão); C) Cobertura 4/91 triturada em gral de ágata e analisada através de microscopia FT-IR; D) Estane 5702-F (transmissão) 
pela técnica PAS ou transmissão, a indicação de algumas bandas do polímero torna-se ainda possível.

\section{Análise ATR IDRIFT-Comparação com os dados obtidos por transmissão}

Na espectroscopia ATR, a radiação que passa através do cristal reflete totalmente na sua superfície interna. Quando um material que absorve, seletivamente, radiação é colocado em contato com um cristal, o feixe penetrará numa camada fina da superfície da amostra e perderá energia naqueles comprimentos de onda, onde o material absorve. A intensidade é atenuada, ou seja, ocorre a refletância total atenuada. Em outras palavras, um espectro de superfície será produzido ${ }^{[16,17]}$. Bom contato entre a amostra e o cristal é essencial ${ }^{[16]}$. Em um experimento DRIFT, a radiação difusamente espalhada é coletada por espelhos e direcionada ao detetor ${ }^{[18]}$. A radiação penetra no interior de uma amostra opaca e retorna após ter sido espalhada várias vezes. As reflexões de superfície e do interior da amostra são medidas ${ }^{[16]}$. Experimentos de refletância externa como os obtidos por DRIFT

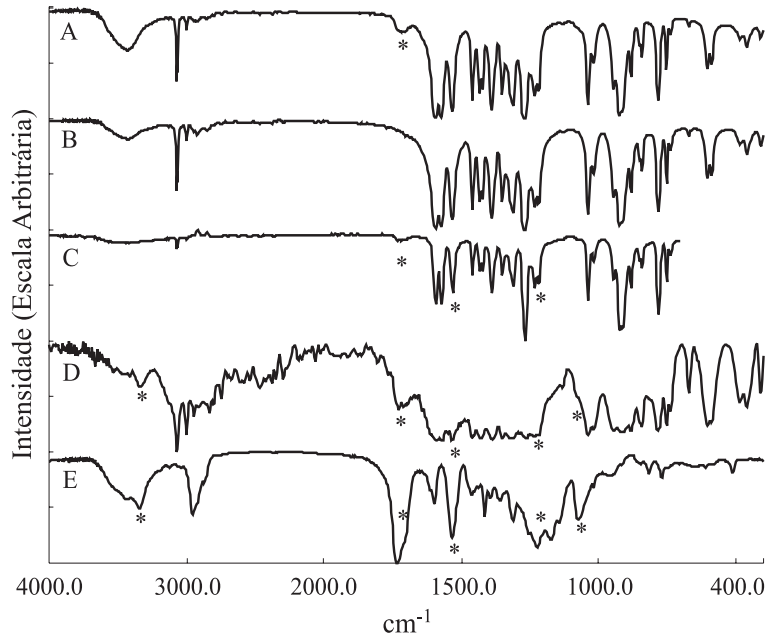

Figura 9. Espectros FT-IR de transmissão e reflexão (DRIFT/ATR) de: A) Cobertura 4/91 (transmissão); B) RDX (transmissão); C) Cobertura 4/91 (ATR); D) Cobertura 4/91 (DRIFT); E) Estane 5702-F (transmissão)

Tabela 2. Principais absorções FT-IR da cobertura RDX/Estane, segundo diferentes técnicas

\begin{tabular}{|c|c|c|c|c|c|c|}
\hline $\begin{array}{c}\text { RDX } \\
\text { (Transmissão) } \\
\text { Espectro de } \\
\text { referência }\end{array}$ & $\begin{array}{c}\text { Estane } \\
\text { (Transmissão) } \\
\text { Espectro de } \\
\text { referência }\end{array}$ & $\begin{array}{r}\text { RDX + Estane } \\
\text { (Transmissão) }\end{array}$ & $\begin{array}{c}\text { RDX + Estane } \\
\text { (MIC) }\end{array}$ & $\begin{array}{c}\text { RDX + Estane } \\
\quad(\text { DRIFT })\end{array}$ & $\begin{array}{c}\text { RDX + Estane } \\
(\text { ATR) }\end{array}$ & $\begin{array}{l}\text { RDX + Estane } \\
\quad \text { (PAS/Pó) }\end{array}$ \\
\hline & $3346(v \mathrm{NH})$ & & 3328 & 3345 & & 3331 \\
\hline \multirow[t]{2}{*}{$\begin{array}{c}3073 \\
\left(v \mathrm{CH}_{2}\right)\end{array}$} & & 3074 & 3071 & 3073 & 3074 & 3074 \\
\hline & $\begin{array}{c}1732 \\
(\nu \mathrm{C}=\mathrm{O})\end{array}$ & 1732 & 1731 & 1728 & 1732 & 1727 \\
\hline \multirow[t]{2}{*}{$1592\left(\mathrm{v}_{\mathrm{s}} \mathrm{NO}_{2}\right)$} & & 1592 & 1614 & 1571 & 1592 & 1591 \\
\hline & $\begin{array}{c}1535 \\
(\delta \mathrm{NH})\end{array}$ & 1532 & 1547 & 1534 & 1531 & 1529 \\
\hline $1459\left(\delta_{\mathrm{s}} \mathrm{CH}_{2}\right)$ & & 1459 & 1506 & 1459 & 1458 & 1571 \\
\hline $1433\left(\delta_{\mathrm{s}} \mathrm{CH}_{2}\right)$ & & 1434 & 1466 & 1431 & 1434 & 1459 \\
\hline $1390\left(\delta_{\mathrm{s}} \mathrm{CH}_{2}\right)$ & & 1392 & 1399 & 1388 & 1388 & 1389 \\
\hline $1351\left(\delta_{\mathrm{s}} \mathrm{CH}_{2}\right)$ & & 1353 & 1358 & 1315 & 1351 & 1351 \\
\hline \multirow[t]{4}{*}{$\begin{array}{c}1270 \\
\left(v_{\mathrm{s}} \mathrm{NO}_{2}+\mathrm{v} \mathrm{N}-\mathrm{N}\right)\end{array}$} & & 1267 & 1289 & & 1267 & 1271 \\
\hline & $\begin{array}{c}1224 \\
(\mathrm{v} \text { C-O)éster }\end{array}$ & 1222 & 1233 & 1231 & 1233 & 1232 \\
\hline & $\begin{array}{c}1174 \\
(v C-O) \text { éster }\end{array}$ & 1178 & & & & \\
\hline & $\begin{array}{c}1076 \\
(\mathrm{vC}-\mathrm{O}) \text { éster }\end{array}$ & 1076 & 1071 & 1074 & & \\
\hline $\begin{array}{c}1039 \\
\text { Vibr. do Anel }\end{array}$ & & 1040 & 1047 & 1038 & 1039 & 1039 \\
\hline \multirow[t]{2}{*}{$\begin{array}{l}\quad 945 \\
\text { Vibr. do Anel }\end{array}$} & & 946 & 951 & & 946 & \\
\hline & & 924 & & & & 921 \\
\hline $\begin{array}{c}604 \\
\left(\tau+\gamma \mathrm{NO}_{2}\right)\end{array}$ & & 604 & & 604 & & 605 \\
\hline
\end{tabular}


apresentam vantagem sobre a refletância interna como ATR, por não existir o problema de contato ótico ${ }^{[17]}$.

No conjunto de espectros da Figura 9 é possível observar que a técnica ATR (espectro C) evidencia, basicamente, as bandas características do RDX (espectro B), e não todas as absorções da espécie polimérica de superfície, como esperado. Isto, provavelmente, possa estar relacionado ao fato que o grau de profundidade de amostragem da técnica e/ou contato promovido entre a amostra e o cristal não serem adequados à análise da espessura do componente polimérico existente nesta amostra.

É válido lembrar que o grau de profundidade de amostragem na técnica ATR varia com o material do prisma e o ângulo utilizado $^{[13]}$.

Embora, possa haver contribuição de componente especular de reflexão em regiões de absorções fortes ${ }^{[19]}$, a análise DRIFT da cobertura 4/91(espectro D), além das bandas de RDX, mostra algumas bandas características do polímero, que está em menor teor $(\sim 5 \%)$. Este resultado, também, está de acordo com as características das técnicas, pois a técnica DRIFT, revela espécies do interior e superfície da amostra.

A Tabela 2 inclui as principais absorções FT-IR da cobertura RDX/Estane, segundo diferentes técnicas. As bandas assinaladas em negrito evidenciam o composto que cada técnica permite observar melhor.

\section{Conclusão}

A aplicação de técnicas FT-IR à caracterização de cobertura de Estane de material energético RDX mostrou que a técnica de transmissão (espectro obtido por pastilha) mostra, basicamente, a presença de RDX e somente sugere a presença de composto polimérico contendo $\mathrm{C}=\mathrm{O}$. Para visualização das bandas da cobertura polimérica, é necessário o tratamento do material com solvente adequado para obtenção de um filme polimérico, e análise pela técnica de transmissão de filme vazado.

Por meio da técnica PAS, é possível identificar o polímero usado no processo de cobertura, e pelas características da técnica PAS, é possível sugerir que este polímero esteja numa camada superficial de poucos micra.

Pode ser observado, que embora o espectro obtido por Microscopia FT-IR da cobertura polimérica, apresente ruídos e não esteja tão bem resolvido quanto os espectros obtidos para a amostra pelas técnicas de transmissão (filme vazado) e PAS, a indicação de algumas bandas do polímero torna-se ainda possível.

A técnica ATR evidencia, basicamente, as bandas características do RDX, e a análise DRIFT também mostra algumas bandas características do polímero.

Embora seja possível observar as bandas do Estane pelas diferentes técnicas na região do infravermelho, as de transmissão (filme vazado) e PAS evidenciam melhor as absorções do poliuretano na amostra analisada de cobertura polimérica de RDX.

Considerando que para obtenção do espectro de transmissão da cobertura 4/91, a preparação da amostra requer tratamento com solvente, e a técnica PAS mostra resultado similar, sem preparação de amostra, pode-se sugerir que esta última técnica seja a mais adequada ao estudo, resultado que pode estar associado à espessura da camada polimérica que a amostra Cobertura 4/91 apresenta.

\section{Agradecimentos}

Os autores agradecem à Engenheira Fabia M. S. Galvão Vialta e sua equipe na pesquisa e no processo de obtenção do RDX coberto com Estane

\section{Referências Bibliográficas}

1. Wanninger, P.; Wild, R.; Kleinschmidt, E.; Späth, H. - "Pressable Explosive Granular Product and Pressad Explosive Charge", U.S. patent 5,547,526 (1996).

2. Benziger, T. M.- "High-Energy Plastic-Bonded Explosive", U.S. patent 3,778,319 (1973).

3. Burgess, C. E.; Woodyard, J. D.; Rainwater, K. A.; Lightfoot, J.M. - "Literature Review of the lifetime of DOE Materials: Aning of Plastic Bonded Explosive and the Explosive and Polymers Contained Therein", Amarilo National Resource Center for Plutonium (1998).

4. Wewerka, E.M.; Loughran, D.E.; Williams, M.J. - "The Effects of Long Term Storage at Elevated Temperatures on Small Cylinders of PBX 9501", Los Almos Scientific Laboratory report, Tech.rept, LA-6302-MS (1976).

5. Urbanski, J. - "Handbook of Analysis of Synthetic Polymers and Plastics", John Wiley \& Sons, New York (1977).

6. Jawhari, T.; Quintanilla, L.; Pastor, J.M. - J. Appl. Polym. Sci., 51, p 463 (1994).

7. Dutra, R.C.L, Takahashi, M.F.K., Diniz, M.F. - Polímeros: Ciência e Tecnologia, 1, p.41 (1995)

8. Dutra, R.C.L.; Soares, B.G.; Campos, E.A., Melo, J.D.G.; Silva, J.L.G. - J. Appl. Polym. Sci., 73, p.69 (1999).

9. Ishida, H.; Rubber Chem. Technol., p. 497 (1987).

10. Mattos, E. C; Dutra, R.C.L.; Diniz, M.F.; David, L.H.; Iha, K.; Anais da Sociedade Brasileira de Química (ABQ), 51, p. 132 (2003).

11. Mattos, E. C; Viganó, I.; Dutra, R.C.L.; Diniz, M.F.; Iha, K.; Quim.Nova, 25, p.722 (2002).

12. Campbell, M.S., M.S., Garcia, D., Idar, D.; Thermochim. Acta, 89 (2000).

13. Smith, A.L.; - "Applied Infrared Spectroscopy”; John Wiley \& Sons; New York (1979).

14. Litch, H.H.; - "HMX (octogen) and its polymorphic forms", in: 2을 Symposium on Chemistry Problems with the Stability of Explosives, Tyringe, Sweden (1970).

15. Chasan, D.E.; Norwitz, G.; Department of the Army, Philadelphia, Tech.rept, T71-6-1 (1971).

16. Pandey, G.C.; Kulshreshtha, A .K. Process Control Qual., 4, p.109 (1993).

17. Graf, R. T.; Koenig, J. L.; Ishida, H.; Polym. Sci. Technol., 36, p. 1 (1987)

18. Urban, M.W.; Prog. Org. Coat., 16, p.321 (1989)

19. Griffiths, P.R.; Haseth, J.A.; -“Chemical Analysis”, John Wiley \& Sons; New York (1986).

Enviado: 05/09/03

Reenviado: $27 / 02 / 04$

Aprovado: 15/03/04 